\title{
Special FX: Harnessing the Farnesoid-X-Receptor to Control Bile Acid Synthesis
}

\author{
Stefano Fiorucci ${ }^{1} \cdot$ Eleonora Distrutti $^{2} \cdot$ Michele Biagioli $^{1}$ \\ Accepted: 10 January 2021 / Published online: 8 February 2021 \\ (C) The Author(s), under exclusive licence to Springer Science+Business Media, LLC part of Springer Nature 2021
}

Bile acids are an heterogeneous group of steroids essential for nutrient absorption and the regulation of metabolism. Cholic acid (CA) and chenodeoxycholic acid (CDCA) are the two most important primary bile acids in humans [1]. CA and CDCA are cholesterol metabolites generated through a chain of 17 enzymatic reactions $[1,2]$ organized in two main metabolic pathways, known as "classic" and "alternative" (Fig. 1). In the classic (or neutral) pathway, the first and rate-limiting enzyme, is the cholesterol- $7 \alpha$-hydroxylase (CYP7A1) that converts cholesterol into $7 \alpha$-hydroxy cholesterol, which is then converted to $7 \alpha$-hydroxy-4cholesten-3-one (C4) by the $3 \beta$-hydroxy- $\Delta^{5}-\mathrm{C}_{27}$-steroid dehydrogenase/isomerase (HSD3B7). The 7 $\alpha$-hydroxy-4cholesten-3-one is then further metabolized by the $\Delta^{4}-3$ oxosteroid $5 \beta$-reductase $(5 \beta$-reductase or AKR1D1) and sterol $12 \alpha$-hydroxylase (CYP8B1) to generate CA [1]. The alternative (or acidic) pathway is initiated by the $\mathrm{C} 27$ hydroxylation of cholesterol by the sterol 27-hydroxylase (CYP27A1) that converts cholesterol to 27-hydroxycholesterol and 3 $\beta$-hydroxy-5-cholestenoic acid, which are then hydroxylated at the $7 \alpha$-position by oxysterol $7 \alpha$-hydroxylase (CYP7B1) [1]. A deficit in the CYP27A1 is the cause of cerebrotendinous xanthomatosis (Fig. 1, red box) a genetic disease characterized by absent/reduced levels of CDCA and tissue deposition of cholesterol/cholestanol and treated by administration of CDCA [4].

As is the case with other steroids, bile acids are signaling molecules activating a family of cell membrane and nuclear receptors termed BAR (bile-activated receptors). The farnesoid-X-receptor (FXR) is the principal bile acid

Stefano Fiorucci

stefano.fiorucci@unipg.it

https://www.gastroenterologia.unipg.it

1 Dipartimento di Medicina E Chirurgia, Università di Perugia, Piazza L. Severi 1, 06100 Perugia, Italy

2 SC di Gastroenterologia Ed Epatologia, Azienda Ospedaliera di Perugia, Perugia, Italy sensor in mammals that is activated in response to increased concentrations of CDCA and CA, which activate FXR at significantly different $\mathrm{EC}_{50}:(\approx 10 \mu \mathrm{M}$ for CDCA and $>50 \mu \mathrm{M}$ for CA).

Bile acid synthesis generates a number of intermediates that accumulate in the liver in the presence of genetic defects of bile acid synthesis, particularly when bile acid homeostasis is impaired by mutations of genes directly involved in the modification of the steroid nucleus. In these settings, a reduction/absence of CA and CDCA not only impairs bile secretion but also prevents FXR activation, promoting the overproduction of hepatotoxic atypical bile acids from intermediates that accumulate in the pathway proximal to the defective enzyme. In the liver, upon binding by CDCA, FXR partners with the retinoid-X-receptor (RXR) to generate a FXR/RXR heterodimer that either directly or indirectly represses the activity of CYP7A1 [7]. FXR regulates the expression/activity of CYP7A1 by at least two distinct, although overlapping, mechanisms (Fig. 1). The first mechanism is a short feedback loop that occurs in hepatocytes and is mediated by the small heterodimer partner (SHP), an atypical nuclear receptor that lacks a DNA binding domain and acts a gene repressor by binding to co-activators or corepressors of target genes [7]. In the case of CYP7A1 and CYP8B1 (Fig. 1), SHP interacts with LRH1 and HNF4 $\alpha$, two positive regulators of the two genes, removing them from a LRH-RE and HFN $4 \alpha-$ RE in the promoter of CYP7A1 and $C Y P 8 B 1$, respectively [7]. The second feedback mechanism is mediated by fibroblast growth factor (FGF) 19, an intestinal FXR-regulated hormone that, once released from ileal epithelial cells (Fig. 1), is transported to the liver through the portal circulation where it binds to the FGF receptor 4 (FGFR4)/ $\beta$-Klotho complex on the sinusoidal membrane of hepatocytes [8]. FGFR4/ $\beta$-Klotho signaling represses the transcription of both $C Y P 7 A 1$ and $C Y P 8 B 1$ but is more active at inhibiting CYP7A1. Since CYP7A1 is important for bile acid homeostasis, it is likely that FXR/ FGF19/FGFR4 signaling represents the major physiological 

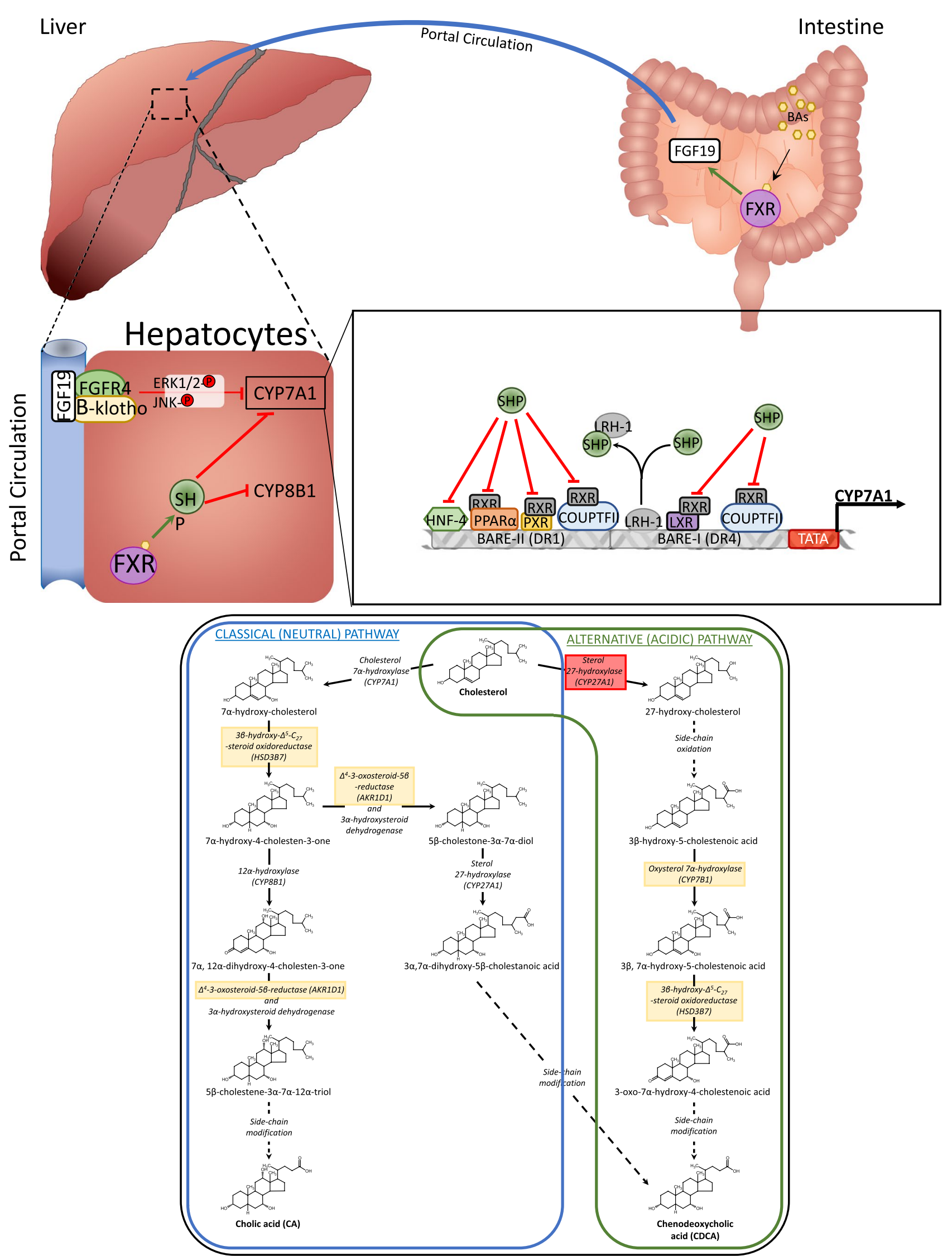
4Fig. 1 Regulation of bile acid synthesis by FXR and its target genes SHP and GFG19. a Schematic of bile acid synthesis. In the liver following chenodeoxycholic acid (CDCA) binding, FXR, a nuclear bile acid sensor, negatively regulates the expression of CYP7A1 and CYP8B1 in a SHP-dependent manner. Once transcribed, SHP binds and removes LRH1 from the promoter of CYP7A1, terminating the synthesis of primary bile acids from cholesterol. Moreover, activation of FXR in the intestine induces the release of fibroblast growth factor (FGF) -19 which reaches the hepatocytes via the portal circulation, binding to FGFR4/ $\beta$-Klotho complex on the cell membrane of hepatocytes. FGFR4 signals to the pERK1-2/pJNK pathway so as to inhibit CYP7A1 activity. b The activation of FXR induces SHP, which inhibits the binding of many transcription factors on BARE-I and BARE-II on the CYP7A1 promoter downregulating the expression of this enzyme. c Bile acid synthesis occurs in hepatocytes via two pathways, termed the classical or neutral pathway and the alternative or acidic pathway, that produce cholic acid (CA) and chenodeoxycholic acid (CDCA). The enzyme responsible for inborn errors in bile acid synthesis identified by Kimoto et al. [9] is highlighted in yellow. Deficiency of CYP27A1, highlighted in red in the figure, is responsible for cerebrotendinous xanthomatosis, a rare autosomal recessive genetic disorder

mechanism for the negative feedback regulation of bile acid synthesis. When bile acid concentrations increase in the liver, the FXR/SHP pathway is also activated in order to prevent further bile acid synthesis [9].

In this issue of Digestive Disease and Sciences, Kimura et al. [9] report a small series of seven Japanese patients with bile acid synthesis disorders caused by homozygous or compound heterozygous loss-of-function mutations of the $3 \beta$-HSD, the $5 \beta$-reductase (AKR1D1), and the oxysterol $7 \alpha$-hydroxylase (Cyp7B1) (Fig. 1, yellow boxes). All three defects impact the modifications of cholesterol nucleus, impairing synthesis of CA and CDCA. The seven patients were identified over 21 years (1996-2017) with diagnosis achieved by measuring atypical bile acids in the blood and urine by fast atom bombardment mass spectrometry (FABMS). The $3 \beta$-HSD deficiency is the most common bile acid disorder in Europe and the USA, accounting for $\approx 40 \%$ of inborn errors of bile acid metabolism in western populations, though it is less prevalent in Japan [9]. On the other hand, the deficiency of oxysterol-7 $\alpha$ hydroxylase (Cyp7B1) is a very rare inborn error of bile acid synthesis, slightly more prevalent in East Asia, that can eventuate in liver failure requiring liver transplantation, although there are reports showing long-term survival with CDCA treatment [2]. The deficiency of CYP7B1 is also associated with neurological defects, emphasizing that the acidic pathway is the major pathway for synthesis of bile acids in infancy and as a means for controlling cholesterol levels in extrahepatic tissues, particularly the nervous system [2]. In general, patients with these three mutations synthesize very little, if any, CA and CDCA, due to their inability to process the cholesterol nucleus (Fig. 1). The deficiency in $3 \beta$-HSD increases the accumulation of $7 \alpha$-hydro-cholesterol (Fig. 1) that is disposed of by generation of atypical $3 \beta$-hydroxy- $\Delta^{5}$ bile acids. These atypical bile acids accumulate in the liver with resultant progressive cholestasis that manifests at ages ranging from a few months to adulthood [2]. The disease is familial and fatal if untreated. A similar pattern of clinical presentations occurs in patients with deficiency of 5 $\beta$-reductase (AKR1D1). The latter disorder manifests in the first few months of life as cholestasis [2] with conjugated hyperbilirubinemia and elevated transaminases, usually progressing to cirrhosis if untreated[10]. Nevertheless, the two diseases respond well to treatment with the exogenous bile acid CA, $5-15 \mathrm{mg} / \mathrm{kg}$, which has been approved for treatment of inborn errors of bile acid metabolism in Western countries, though CDCA and a combination of CDCA and UDCA have also been proven effective in reducing the production of atypical bile acids [6]. As mentioned above both CA and CDA are FXR ligands, whereas UDCA is neutral toward the receptor. In this Japanese series [9], three patients with $3 \beta$-HSD deficiency and two patients with $5 \beta$-reductase deficiency were administered CDCA due to a lack of availability of CA in Japan. The patient with oxysterol $7 \alpha$ hydroxylase deficiency underwent a liver transplantation that was curative, with no evidence of liver fibrosis 10 years after the procedure. One patient with $5 \beta$-reductase (AKR1D1) deficiency was administered UDCA only for the first 12 months of life due to the parents refusing further therapy; the patient was healthy at 16-year follow-up. The doses of CDCA used by Kimura et al. [9] ranged from 4 to $7.8 \mathrm{mg} / \mathrm{kg} /$ day with the median duration of therapy 10 years. CDCA treatment improved hepatic biochemistries with follow-up imaging showing no evidence of liver cirrhosis, liver tumor, or biliary stones. Transient elastography performed in all patients, but two showed no fibrosis during the follow-up period. Of relevance, analysis of bile acids profiles demonstrated that CDCA treatment decreased the serum and urinary concentrations of unusual bile acids: $3 \beta$-hydroxy- $\Delta^{5}$ bile acids and 3-oxo- $\Delta^{4}$ bile acids in serum and urine ranged from 2.4 to $25.1 \mu \mathrm{mol} / \mathrm{L}$ and 16.3 to $123.9 \mu \mathrm{mol} / \mathrm{mmol}$ creatinine, respectively, before treatment and decreased to $0.1-5.7 \mu \mathrm{mol} / \mathrm{L}$ and $2.0-13.9 \mu \mathrm{mol} /$ mmol creatinine, respectively, indicating a repression of endogenous bile acid synthesis (CDCA became the major bile acid detectable in the serum of all five patients, slightly increasing total bile acid concentrations). Because CDCA is a FXR ligand and FXR regulates the expression of CYP7A1 as mentioned above [4], the data reported by Kimura et al. [9] are consistent with activation of a FXR-dependent mechanism. Unfortunately, there were no data regarding FGF19, which would have helped to understand the functionality of intestinal FXR in these patients. [...].

The data presented by Kimura et al. [9] demonstrate that FXR activation rescues inborn errors of bile acid metabolism, most likely exploiting the aforementioned repression of CYP7A1. This report, therefore, raises the question of using potent semisynthetic or synthetic FXR ligands in these 
clinical settings. Though obeticholic acid is a significantly more potent FXR agonist than CDCA and CA, currently approved for clinical use in patients with primary biliary cholangitis (PBC) $[1,5,3]$, other synthetic FXR ligands are currently under development, including tropixefor, nidufexor, and cilofexor among others [5,3]. Whether these novel FXR agonists will add to the beneficial effects of CA or CDCA in patients with inborn errors of bile acid metabolism is unclear though worthy of investigation. Another interesting thought that arises from the Kimura's paper is that CDCA therapy did not cause pruritus, a common side effect of FXR agonism of unclear mechanism. The fact that CDCA did not cause pruritus questions the primary function of FXR activation in mediating the beneficial effects of CDCA, thus suggesting that other receptors might be involved.

In summary, the study of Kimura et al. demonstrates that therapy with exogenous bile acids can slow or even halt the progression of liver disease in children born with bile acid synthesis disorders, presumably based on the ability of these bile acids to activate FXR, the nuclear bile acid receptor. Whether related diseases are also amenable to this type of therapy and whether partially or fully synthetic FXR agonists are therapeutic are questions to be answered by further studies of this nature.

\section{References}

1. Chiang JY. Bile acid metabolism and signaling. Compr Physiol 2013;3:1191-1212. https://doi.org/10.1002/cphy.c120023.
2. Clayton PT. Disorders of bile acid synthesis. J Inherit Metab Dis 2011;34:593-604. https://doi.org/10.1007/s10545-010-9259-3.

3. Fiorucci S, Distrutti E. The pharmacology of bile acids and their receptors. Handb Exp Pharmacol 2019;256:3-18. https://doi. org/10.1007/164_2019_238.

4. Fiorucci S, Distrutti E. Chenodeoxycholic acid: an update on its therapeutic applications. Handb Exp Pharmacol 2019;256:265282. https://doi.org/10.1007/164_2019_226.

5. Fiorucci S, Di Giorgio C, Distrutti E. Obeticholic acid: an update of its pharmacological activities in liver disorders. Handb Exp Pharmacol 2019;256:283-295. https://doi. org/10.1007/164_2019_227.

6. Gonzales E, Matarazzo L, Franchi-Abella S et al. Cholic acid for primary bile acid synthesis defects: a life-saving therapy allowing a favorable outcome in adulthood. Orphanet J Rare Dis 2018;13:190. https://doi.org/10.1186/s13023-018-0920-5.

7. Goodwin B, Jones SA, Price RR et al. A regulatory cascade of the nuclear receptors FXR, SHP-1, and LRH-1 represses bile acid biosynthesis. Mol Cell 2000;6:517-526. https://doi.org/10.1016/ s1097-2765(00)00051-4.

8. Inagaki T, Choi M, Moschetta A et al. Fibroblast growth factor 15 functions as an enterohepatic signal to regulate bile acid homeostasis. Cell Metab 2005;2:217-225. https://doi.org/10.1016/j. cmet.2005.09.001.

9. Kimura A, Mizuochi T, Takei $\mathrm{H}$ et al. Bile acid synthesis disorders in Japan: long-term outcome and chenodeoxycholic acid treatment. Dig Dis Sci. (Epub ahead of print). https://doi.org/10.1007/ s10620-020-06722-4.

10. Yanagi T, Mizuochi T, Homma K et al. Distinguishing primary from secondary $\Delta(4)$-3-oxosteroid $5 \beta$-reductase (SRD5B1, AKR1D1) deficiency by urinary steroid analysis. Clin Endocrinol (Oxf) 2015;82:346-351. https://doi.org/10.1111/cen.12596.

Publisher's Note Springer Nature remains neutral with regard to jurisdictional claims in published maps and institutional affiliations. 